\title{
The Solubility of Organic Compounds in Supercritical $\mathrm{CO}_{2}$
}

\author{
Guillermo A. Alvarez ${ }^{\mathrm{a}}$, Wolfram Baumann ${ }^{\mathrm{b}}$, Martha Bohrer Adaime ${ }^{\mathrm{b}}$, and Frank Neitzel ${ }^{\mathrm{b}}$ \\ a Departamento de Química, Facultad de Ciencias, Universidad de los Andes, \\ Carrera 1 No. 18A-10/70, Bogotá, Colombia \\ ${ }^{\mathrm{b}}$ Institute of Physical Chemistry, University of Mainz, D-55099 Mainz, Germany \\ Reprint requests to Prof. G. A. A.; Fax: 00571 3324366; E-mail: gualvare@ uniandes.edu.co
}

Z. Naturforsch. 60a, $641-648$ (2005); received May 3, 2005

A simple liquid solution model is proposed to describe the effect of solvent-solute interactions on the solubility of nonpolar and slightly polar substances in supercritical solvents. Treating the system as an ideal solution, the effect of pressure on the solubility is zero or nearly zero, as it is governed by the difference in molar volume of the pure supercooled liquid solute and the pure solid solute, and this may be nearly zero. Deviations from ideal behavior are given by activity coefficients of the Margules type with the interaction parameter $w$ interpreted as interchange energy as in the lattice theory. The hypothesis is put forward that the interchange energy is of the same form as a function proposed by Liptay and others to describe the effect of the solvent on the wavelength of the absorption maximum of the solute dissolved in the solvent. The function consists of a radius of interaction $a$ and a function $g(\varepsilon)$ of the dielectric constant $\varepsilon$ of the solvent, treated as a continuum. The function $g$ depends on pressure through the pressure dependence of the dielectric constant $\varepsilon(P)$. The attractive feature of this formalism, introduced by Baumann et al. and here justified thermodynamically, is that plots of the logarithm of solubility vs. $g$ are linear, except for polar solutes near the solvent's critical point. Changes in slope then admit interpretation as changes in the radius of interaction $a$ with possible clues about the mechanism of solvation of these molecules.

Key words: Dielectric Interactions; Solubility; Supercritical $\mathrm{CO}_{2}$; Thermodynamic Model; Activity Coefficient.

\section{Introduction}

The solubility of substances in supercritical solvents is relevant in separation processes such as supercritical fluid extraction and chromatography and for modeling the state of a solute molecule in a solvent [1]. In particular, since near the critical point the solvent's density can change by a factor of ten with only small changes in the temperature and the pressure, it is clear that the solubility of the solute can vary likewise by a large factor. Enormous changes in the nature of the solvent, quantified for example by the interchange energy $w$ of the lattice theory, would be required to bring about the simple "distance" effect due to the compressibility of the solvent near the critical point.

It thus appears that the solvent's density for a solvent near the critical point is a suitable measure of solventsolute interaction and hence of solubility. Accordingly, plots of the logarithm of solubility vs. the density or the logarithm of the density obey a linear relationship in many cases [2]. Since the dielectric constant of the solvent depends nearly linearly on the density [3], one may substitute the dielectric constant $\varepsilon(P)$ by the density $\rho(P)$ as a measure of solvent-solute interactions in practical cases.

However, we can proceed further according to the theories of Lippert [4], Mataga et al. [5] and notably Liptay [6], who showed that in polar solvents the change due to the solvent in the wavelength at the maximum of absorption of light by the solute or solvatochromic shift is a function of $(\varepsilon(P)-1) /$ $\left(a^{3}(2 \varepsilon(P)+1)\right)=g(\varepsilon) / a^{3}$. Further, the shift in solution is towards red relative to the gas phase, that is the solvent-solute system is stabilized by dispersion interactions and by interactions of the dipole of the molecule with the surrounding dielectric. Since the function $\varepsilon(P)$ increases monotonically with $P$, this stabilizing effect for constant $a$ always increases the solubility, reaching an asymptotic level at high $P$ (but see below). Thus, here the interchange energy is negative, leading to negative deviations of Raoult's law, in contrast to the usual positive deviations for nonpolar solutes in nonpolar solvents. 
Numerous models have been proposed for the effect of the thermodynamic variables temperature $T$ and pressure $P$ on the mole fraction $x_{\mathrm{B}}$ of solute $\mathrm{B}$ in the saturated solution since the work of Schröder on the effect of temperature on the solubility of naphthalene in benzene (see e.g. Pitzer [7]). The present formalism follows the same steps of Schröder but considering changes of pressure instead of changes of temperature. Whereas molar enthalpy is the variable that controls ideal solubility as a function of temperature, here molar volume controls ideal solubility as a function of pressure. In contrast to the temperature effect, the ideal pressure effect is, however, very small or zero, the changes in solubility arising from real interactions between solvent and solute.

The model of Kurnik and Reid [8] is possibly one of the most comprehensive models of solubility of solids or liquids in compressed gases. Using volumetric data at constant temperature and composition and some mixing rules, they predict ideal solubility initially decreasing with pressure, passing through a minimum, rising dramatically again as the vapor phase goes through the critical point and, apparently, going through a maximum at very high pressure and decreasing solubility thereafter due to "repulsive forces" between solute and solvent. The steep rise in solubility near the critical point is attributed to real attractive interactions as evidenced by a vapor-phase fugacity coefficient in the high-pressure gas mixture, which is much less than one. The present model, starting from an ideal liquid solution and describing deviations from ideal behavior through an energy parameter suggested by the work of Liptay [6] may be considered a simplification of the model of Kurnik and Reid [8]; both models attribute enhanced solubility near the critical point to real attractive interactions between solute and solvent. The present model cannot predict the minimum in solubility reported by Kurnik and Reid [8] (which may be of commercial importance, for example if a stream of gas is to be kept as free as possible of impurities; but see discussion below) and as mentioned above, neither can the maximum reported by them be predicted, as the type of forces responsible for the solvatochromic shift are always attractive. A refined model, which takes into account association of polar solutes for large values of the interaction parameter and/or large solute concentration, is explained below the discussion. This effect may contribute or eventually account for the mentioned maximum.

\section{Thermodynamic Model}

It is often the case for solutes with high melting points that solid B dissolves in liquid A at a given pressure only up to a certain mole fraction $x_{\mathrm{B}}$ called the equilibrium mole fraction or solubility of the solute in the solution saturated with the solid. For component B, equilibrium between a solid phase $\mathrm{s}$ and a liquid phase 1 is dictated by the equality of chemical potentials

$$
\mu_{\mathrm{B}}^{\mathrm{s}}=\mu_{\mathrm{B}}^{1}
$$

The chemical potential of the pure solid phase B is

$$
\mu_{\mathrm{B}}^{\mathrm{s}}=\mu_{\mathrm{B}}^{0 \mathrm{~s}}
$$

where the upper index 0 denotes a pure phase. For the component $\mathrm{B}$ in the mixed phase,

$$
\mu_{\mathrm{B}}^{1}=\mu_{\mathrm{B}}^{* 1}(T, P)+R T \ln a_{\mathrm{B}} .
$$

$\mu_{\mathrm{B}}^{* 1}(T, P)$ is the chemical potential of the supercooled pure liquid $\mathrm{B}$ with the dependencies on temperature and pressure indicated explicitly. $a_{\mathrm{B}}$ is the activity of component $\mathrm{B}$ in solution and $R$ is the universal gas constant.

Replacing (2) and (3) in (1) gives:

$$
\mu_{\mathrm{B}}^{0 \mathrm{~s}}=\mu_{\mathrm{B}}^{* 1}+R T \ln a_{\mathrm{B}}
$$

Consider the effect of pressure on activity at $T=$ constant. Taking derivatives with respect to pressure

$$
\left(\frac{\partial \mu_{\mathrm{B}}^{0 \mathrm{~s}}}{\partial P}\right)_{T}=\left(\frac{\partial \mu_{\mathrm{B}}^{* 1}}{\partial P}\right)_{T}+R T\left[\frac{\partial\left(\ln a_{\mathrm{B}}\right)}{\partial P}\right]_{T} .
$$

For a pure substance

$$
\mathrm{d} \mu_{\mathrm{B}}^{0}=\left(\frac{\partial \mu_{\mathrm{B}}^{0}}{\partial P}\right)_{T} \mathrm{~d} P+\left(\frac{\partial \mu_{\mathrm{B}}^{0}}{\partial T}\right)_{P} \mathrm{~d} T,
$$

where

$$
\left(\frac{\partial \mu}{\partial P}\right)_{T}=V_{\mathrm{m}}
$$

and

$$
\left(\frac{\partial \mu}{\partial T}\right)_{P}=-S_{\mathrm{m}}
$$

$V_{\mathrm{m}}$ and $S_{\mathrm{m}}$ are the molar volume and the molar entropy, respectively, of the pure substance. For a process at 
constant temperature the second term on the right in (6) vanishes. Substituting (7) in (5) yields:

$$
V_{\mathrm{m}}^{0 \mathrm{~s}}=V_{\mathrm{m}}^{* 1}+R T\left[\frac{\partial\left(\ln a_{\mathrm{B}}\right)}{\partial P}\right]_{T}
$$

or rearranging

$$
\left[\frac{\partial\left(\ln a_{\mathrm{B}}\right)}{\partial P}\right]_{T}=\frac{V_{\mathrm{m}}^{0 \mathrm{~s}}-V_{\mathrm{m}}^{* 1}}{R T}=-\frac{\Delta_{\text {fusion }} V_{\mathrm{m}}}{R T},
$$

where $\Delta_{\text {fusion }} V_{\mathrm{m}}$ is the change in molar volume on melting the solid to the supercooled liquid. Presumably, $\Delta_{\text {fusion }} V_{\mathrm{m}}$ is a positive quantity, so that the slope in (10) is negative and the activity of the solute decreases with an increase in pressure at constant temperature. Taking the liquid and solid substances as incompressible over a limited pressure range, the quantity $\Delta V_{\mathrm{m}}$ is a constant, and a simple integration gives

$$
\begin{aligned}
& \int_{\ln a_{\mathrm{B}}^{\prime}}^{\ln a_{\mathrm{B}}^{\prime \prime}} \mathrm{d}\left(\ln a_{\mathrm{B}}\right)=-\frac{\Delta_{\text {fusion }} V_{\mathrm{m}}}{R T} \int_{P^{\prime}}^{P^{\prime \prime}} \mathrm{d} P, \\
& T=\text { const. }
\end{aligned}
$$

This equation is entirely analogous to the Schröder equation for solubility as a function of temperature [7]. Using $P^{*}$ as the pressure of the pure supercooled liquid at $T$ we obtain

$$
\ln a_{\mathrm{B}}=-\frac{\Delta_{\text {fusion }} V_{\mathrm{m}}}{R T}\left(P-P^{*}\right),
$$

and this may be written

$$
\ln a_{\mathrm{B}}=c_{0}-\frac{\Delta_{\text {fusion }} V_{\mathrm{m}}}{R T} P
$$

where $c_{0}$ is a constant.

\section{The Activity Coefficient}

For an ideal solution $a_{\mathrm{B}} \approx x_{\mathrm{B}}$, the mole fraction of $\mathrm{B}$ in solution. Then (13) becomes

$$
\ln x_{\mathrm{B}}=c_{0}-\frac{\Delta_{\text {fusion }} V_{\mathrm{m}}}{R T} P .
$$

With $\Delta_{\text {fusion }} V_{\mathrm{m}}>0$, this equation is reminiscent of Kurnik and Reid's result [8] that solubility in the ideal gas decreases with an increase in pressure. On the other hand, for a typical solid or liquid molar volume of $10^{-4} \mathrm{~m}^{3}$ and a volume change of melting of $10 \%$ of the volume, the factor $\Delta V_{\mathrm{m}} / R T \approx 4 \cdot 10^{-9} \mathrm{~Pa}^{-1}$ at
$T=300 \mathrm{~K}$. This leaves ideal solubility practically independent of pressure up to say $30 \mathrm{MPa}$.

For a real solution $a_{\mathrm{B}}=\gamma_{\mathrm{B}} x_{\mathrm{B}}$, where $\gamma_{\mathrm{B}}$ is the activity coefficient of the solute $\mathrm{B}$ in the solution. Replacing this in (13) and neglecting the $\Delta_{\text {fusion }} V_{\mathrm{m}}$ term gives

$$
\ln x_{\mathrm{B}}=c_{0}-\ln \gamma_{\mathrm{B}}
$$

For the activity coefficient we adopt the Margules equation

$$
\ln \gamma_{\mathrm{B}}=\frac{w}{k T} x_{\mathrm{A}}^{2}
$$

$x_{\mathrm{A}}$ is the mole fraction of solvent $\mathrm{A}$ and $k$ is Boltzmann's constant. In the original Margules equation $w$ is an energy parameter; in the lattice theory $w$ is the interchange energy given by

$$
w=z\left[\Gamma_{\mathrm{AB}}-\frac{1}{2}\left(\Gamma_{\mathrm{AA}}+\Gamma_{\mathrm{BB}}\right)\right],
$$

where $\Gamma_{\mathrm{AB}}$ is the potential energy of an $(\mathrm{A}-\mathrm{B})$ pair and likewise $\Gamma_{\mathrm{AA}}$ and $\Gamma_{\mathrm{BB}}$ of $(\mathrm{A}-\mathrm{A})$ and $(\mathrm{B}-\mathrm{B})$ pairs, respectively. $z$ is the coordination number of the lattice. Physically, $w$ is the energy absorbed in the process of separating $z$ pairs of type (A-A) and $z$ pairs of type (B-B) to form $2 z$ dissimilar (A-B) pairs. Calculations of $w$ from molecular properties are due to Kohler using London's theory of dispersion forces. Inadequate knowledge of intermolecular forces hampers this type of calculation [1].

Liptay [6], and also Lippert [5] and Mataga et al. [6], obtained an expression for the effect of the solvent on the wavenumber of absorption of the dissolved molecule. The main part of this calculation is the estimation of the energy of the dissolved molecule relative to its energy in the gas phase; while the change in energy is attributed to the dissolved molecule, necessarily both solute and surrounding solvent molecules are involved in this change. For the estimation of the energy, the model of Onsager [9] is used, according to which the molecule is located at the center of a cavity within an isotropic and homogeneous dielectric medium, which represents the solvent. Under the assumption that the permanent dipole of the molecule in the ground state $\mu_{\mathrm{g}}^{0}$ is parallel to the permanent dipole of the molecule in the given (Franck-Condon) excited state $\mu_{\mathrm{a}^{\prime}}^{0}$ and neglecting polarizability terms, the final result for the wavenumber $\tilde{v}_{\mathrm{a}}$ of the absorption maximum of the molecule dissolved in an unpolar solvent 
is [10]

$$
h c \tilde{v}_{\mathrm{a}}=\text { const. }-f\left(h c D+\frac{1}{2}\left(\mu_{\mathrm{a}^{\prime}}^{0}\right)^{2}-\frac{1}{2}\left(\mu_{\mathrm{g}}^{0}\right)^{2}\right) .
$$

$h$ is Planck's constant, $c$ the speed of light, and the term $-f h c D$ represents the difference in dispersion interactions between ground and excited states. $D$ is neglected for polar molecules. $f$ is a positive quantity required in the description of the cavity. For a spherical cavity of radius $a$ and the dipole moment of the molecule represented by a point dipole at the center of the cavity, one has

$$
f=\frac{1}{4 \pi \varepsilon_{0}} \frac{2}{a^{3}} \frac{\varepsilon-1}{2 \varepsilon+1}=\frac{1}{2 \pi \varepsilon_{0} a^{3}} g,
$$

where $\varepsilon_{0}$ is the permittivity of vacuum.

The negative sign in front of $f$ in (18) leads to the red shift toward lower energy from the gas to the dissolved molecule for the usual case, where $\mu_{\mathrm{a}^{\prime}}^{0} \geq \mu_{\mathrm{g}}^{0}$. The validity of (18) was tested meticulously by Liptay [6], who plotted $\tilde{v}_{\mathrm{a}}$ against $g$ for many solvents of known $\varepsilon$ and obtained excellent straight lines.

Noting that $h c \tilde{v}_{\mathrm{a}}$ represents the energy of interaction of the solute with the solvent, Rodrigues et al. [11] and Neitzel [10] began to plot log solubility vs. $g$ and obtained very good straight lines in many cases. Formally then, we adopt the following form for the exchange energy based on (18):

$$
w=c_{1}-\frac{c_{2}}{a^{3}} g
$$

and this may be substituted in (15) with (16), redefining $c_{1}$ and $c_{2}$ for constant $T$ and $x_{\mathrm{B}} \rightarrow 0$ :

$$
\ln x_{\mathrm{B}}=c_{0}+\frac{c_{1}}{a^{3}} g
$$

This is the equation used in [10] to correlate a relatively extensive series of experimental results. If needed, the temperature which appears in (16) may be passed explicitly onto this equation to correlate data at different temperatures. A brief summary of the results follows.

\section{Results and Discussion}

In order to demonstrate the applicability of the proposed model, data from [10] and [2] are used which have carefully been read from the published graphs. The data in [2] are taken directly as mole fraction of

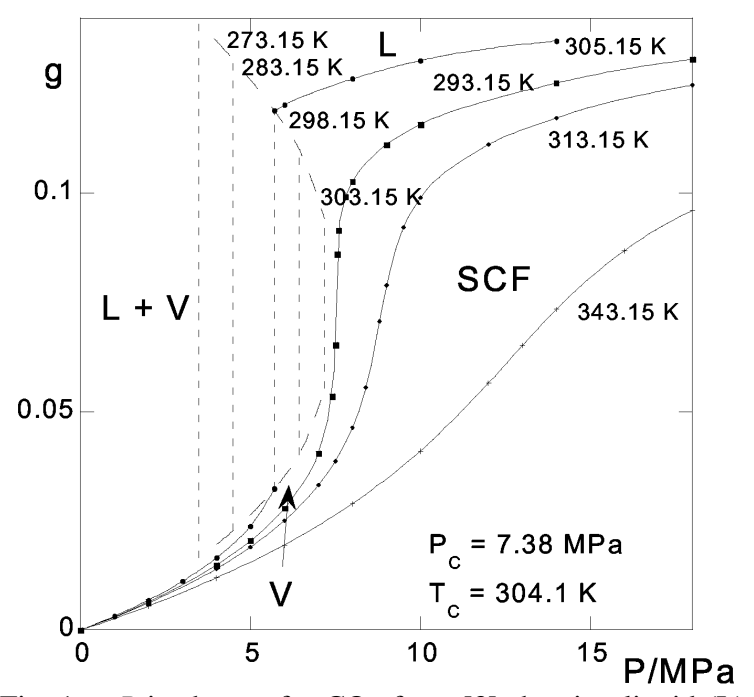

Fig. 1. $g-P$ isotherms for $\mathrm{CO}_{2}$ from [3] showing liquid (L), vapor $(\mathrm{V})$, coexisting liquid and vapor $(\mathrm{L}+\mathrm{V})$ and supercritical fluid (SCF) regions. $P_{\mathrm{C}}=7.38 \mathrm{MPa}, T_{\mathrm{C}}=304.1 \mathrm{~K}$.

B. The data in [10] are given in units of mmol of $B$ per Liter, $c_{\mathrm{B}}$, and were converted to mole fraction of $\mathrm{B}, x_{\mathrm{B}}$, in the following way [10]:

$$
x_{\mathrm{B}}=\frac{n_{\mathrm{B}}}{n_{\mathrm{B}}+n_{\mathrm{A}}} \approx \frac{n_{\mathrm{B}}}{n_{\mathrm{A}}}=\frac{\frac{n_{\mathrm{B}}}{V}}{\frac{n_{\mathrm{A}}}{V}}=\frac{c_{\mathrm{B}}}{\rho_{\mathrm{A}}},
$$

$n_{\mathrm{B}}$ is the number of moles of $\mathrm{B}, n_{\mathrm{A}}$ is the number of moles of $\mathrm{A}$ and $\rho_{\mathrm{A}}$ is the molar density of solvent $\mathrm{A}$ in $\mathrm{mmol} / \mathrm{l}$ at the given pressure. The $c_{\mathrm{B}}$ data in [10] were measured spectroscopically from a reference concentration using Lambert-Beer's law.

Two solvents, carbon dioxide, which is an unpolar molecule, and trifluoromethane, which is a slightly polar solvent were investigated in [10]. $\varepsilon-\rho-P$ isotherms for $\mathrm{CO}_{2}$ were taken from [3]; for $\mathrm{CF}_{3} \mathrm{H}, \varepsilon-P$ isotherms over the whole range and $\varepsilon$ - $\rho$ isotherms from 0 to $100 \mathrm{~kg} \mathrm{~m}^{-3}$ were found in [12]. A long extrapolation up to $700 \mathrm{~kg} \mathrm{~m}^{-3}$ was then made of the latter data with the help of the Clausius-Mossotti equation. This was necessary to obtain the $\rho-P$ isotherm for the conversion of the data in [10] to mole fraction according to (22); it was verified that the result of the extrapolation within reasonable limits has negligible effect on the results shown below (see Fig. 5).

It is instructive to consider first the $g-P$ isotherms for $\mathrm{CO}_{2}$ (Fig. 1) as the underlying cause of the phenomena of solubility investigated here. Over the pressure range from 5 to $16 \mathrm{MPa}$ at $T=313.15 \mathrm{~K}$, where most of the data lie, $g_{\mathrm{CO}_{2}}$ increases almost tenfold. As the fluid 


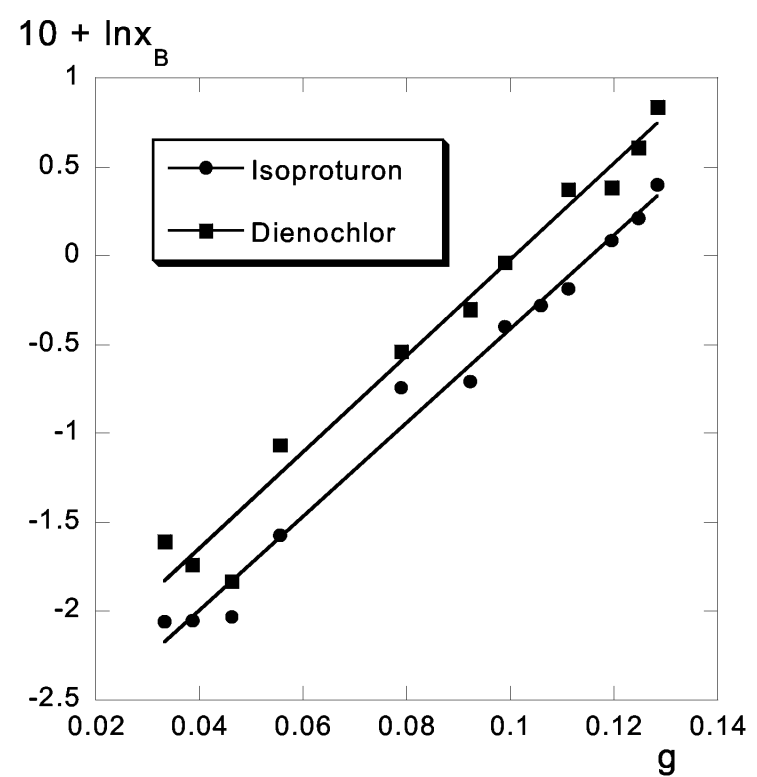

Fig. 2. Ln solubility vs. $g$ for the nonpolar pesticides isoproturon and dienochlor in superfluid carbon dioxide at $T=$ $313.15 \mathrm{~K}$.

approaches incompressibility the isotherms of $g$ vs. $P$ level off. The similarity with $\rho-P$ isotherms is apparent but the increase in $\rho$ is only about eightfold at this temperature.

The simplest data to analyze are nonpolar less volatile solutes such as the pesticides isoproturon and dienochlor in the nonpolar solvent carbon dioxide. In Fig. 2 the $\ln x_{\mathrm{B}}$ vs. $g$ experimental data points are very well approximated by straight regression lines with correlation coefficients $r=0.993$ and 0.987 , respectively.

To demonstrate the correctness of the procedure employed in Fig. 2, the same solubility data are replotted in Fig. 3 against pressure along with the solubility predicted by the linear regression of Figure 2 . In a remarkable way, the regression line of Fig. 2 reproduces the complex S-shaped experimental curve practically exactly. Note that, plotted in this fashion, the present S-shaped curve bears resemblance to the similarly S-shaped curve of Kurnik and Reid [8]. In the same spirit, one may extrapolate the regression line toward zero pressure until it meets the ideal gas law [1]

$$
x_{\mathrm{B}}=\frac{P_{\mathrm{B}}^{\mathrm{s}}}{P}
$$

where $P_{\mathrm{B}}^{\mathrm{s}}$ is the saturation (vapor) pressure of pure solid $\mathrm{B}$ and $P$ is the pressure. This would give an idea

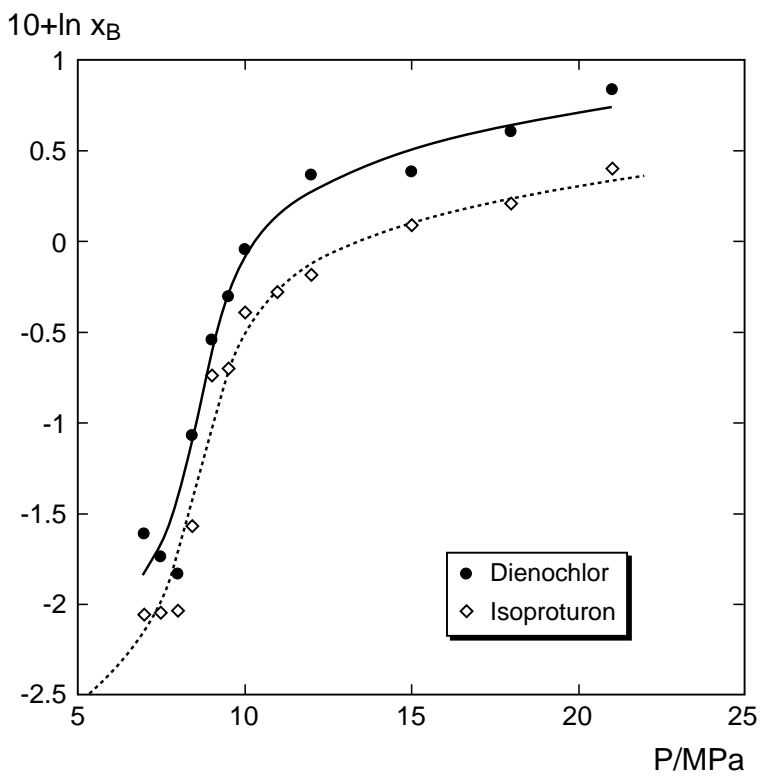

Fig. 3. Ln solubility vs. pressure of the isoproturon and dienochlor data in Fig. 2, with the regression lines of Fig. 2 replotted in this graph.

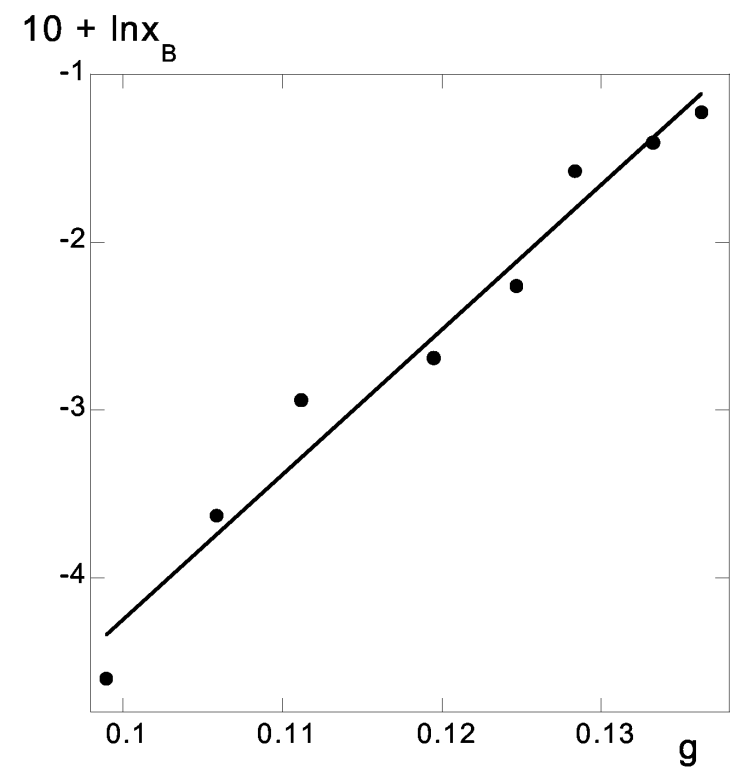

Fig. 4. Ln solubility vs. $g$ for the slightly polar donor-acceptor molecule 5-dimethylamino-5'-nitro-2,2'-bithiophene in superfluid carbon dioxide at $T=313.15 \mathrm{~K}$ showing normal linear behavior in this plot.

of the minimum in solubility mentioned in the introduction.

Similar results are obtained for polar nonvolatile molecules such as the donor-acceptor conjugated 5- 


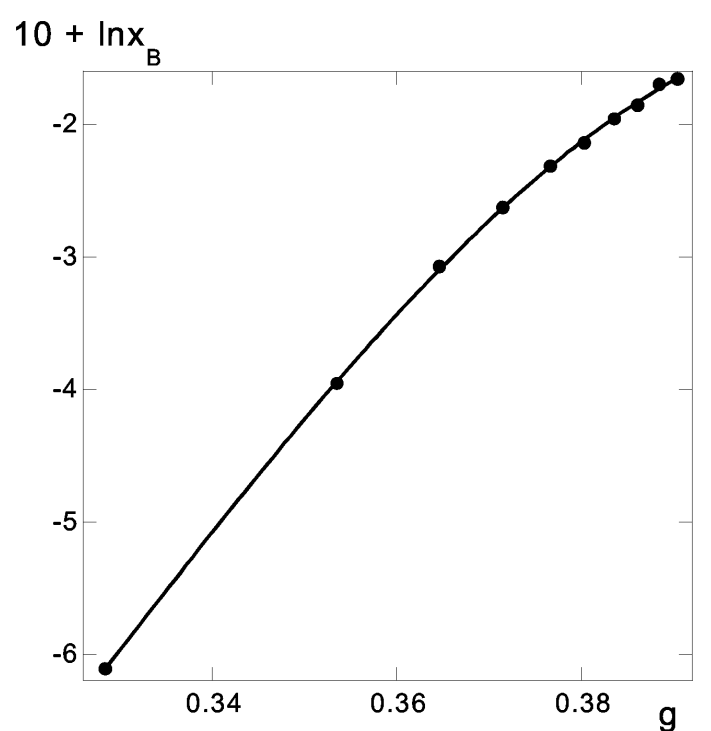

Fig. 5. Ln solubility vs. $g$ for the donor-acceptor molecule in Fig. 4 dissolved in the slightly polar fluid trifluoromethane at $T=313.15 \mathrm{~K}$ showing some deviation from the linear relation proposed here assuming the equation of state data for the solvent are correct.

dimethylamino-5'-nitro-2,2'-bithiophene in the nonpolar supercritical carbon dioxide solvent, as shown in Figure 4. A good straight line is obtained again, the increased scatter being attributed to experimental detection difficulties due to the lower solubility of the polar solute in the nonpolar solvent. The slope of this plot is 86.4 in contrast to the lesser slopes of 26.4 and 27.1 reported for the nonpolar solutes isoproturon and dienochlor in Fig. 2 in accord with the assumption made that this slope contains molecular quantities such as dipole moments and polarizabilities, which describe the solubility.

To improve the solubility of the polar molecule 5dimethylamino-5'-nitro-2,2'-bithiophene a slightly polar solvent, trifluoromethane, was used. The plot of $\ln$ solubility vs. $g$ at $T=313.15 \mathrm{~K}$ is no longer linear as shown in Fig. 5, assuming the $\varepsilon-\rho-P$ data are correct.

Similarly, the respective plot of $\ln x_{\mathrm{B}}$ versus $g$ for more volatile compounds is not linear, as is shown in Fig. 6 for three polyaromatic hydrocarbons, naphthalene at $328.15 \mathrm{~K}$ from Spiliotis et al. [13] and for phenanthrene and phenylanthracene at $313.15 \mathrm{~K}$ from [10].

\section{A More Refined Theory}

A modified theory known as the quasichemical approximation was introduced by Guggenheim (see [1]) to account for ordering effects for example when polar molecules are dissolved in nonpolar solvents without change of volume. The polar solute molecules will tend to surround themselves with other solute molecules in favor of the solvent-solute contacts thus lowering solubility. This may explain the downturn in the straight line observed at high $g$ for the polar solutes e.g. 5dimethylamino-5' -nitro-2,2'-bithiophene in Figure 5.

An equilibrium constant $K$ is defined for the "reaction"

$$
(\mathrm{A}-\mathrm{A})+(\mathrm{B}-\mathrm{B})=2(\mathrm{~A}-\mathrm{B})
$$

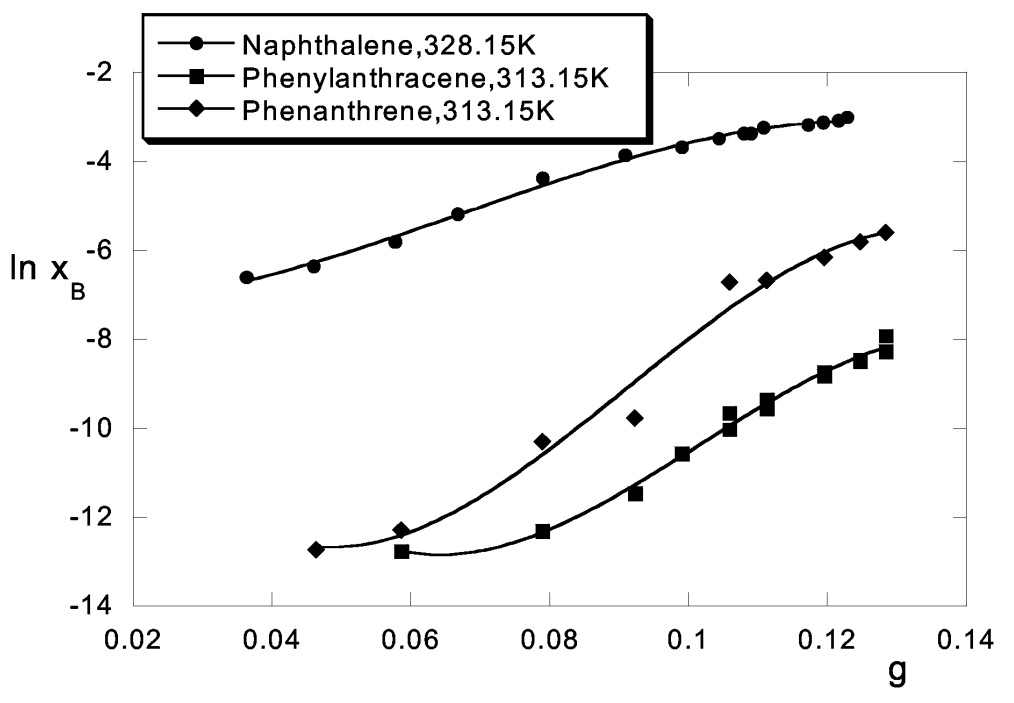

Fig. 6. Ln solubility vs. $g$ for some polyaromatic hydrocarbons in $\mathrm{CO}_{2}$ at $T=$ $313.15 \mathrm{~K}$. The regression curve is calculated according to the cubic equation (33). 
Setting

$$
\Delta_{\mathrm{r}} u=N_{\mathrm{A}} \frac{2 w}{z}
$$

for the molar energy change of the "reaction" with $N_{\mathrm{A}}$ Avogadro's number and this in the van't Hoff equation, integrating at constant volume and using some conservation relations gives for the excess energy of mixing $u^{\mathrm{E}}$

$$
u^{\mathrm{E}}=u^{\mathrm{E}^{*}}\left(\frac{2}{\beta+1}\right)
$$

where $u^{\mathrm{E}^{*}}$ is the excess energy of the random mixture

$$
u^{\mathrm{E}^{*}}=N_{\mathrm{A}} w x_{\mathrm{A}} x_{\mathrm{B}}
$$

and the parameter $\beta$ is:

$$
\beta=\left[1+4\left(\mathrm{e}^{2 w / z k T}-1\right) x_{\mathrm{A}} x_{\mathrm{B}}\right]^{1 / 2} .
$$

The excess Helmholtz and excess Gibbs energies are obtained by integrating the Gibbs-Helmholtz equation at constant volume $\left(v^{\mathrm{E}}=0\right)$ with the result [1]:

$$
\frac{a^{\mathrm{E}}}{R T}=\frac{g^{\mathrm{E}}}{R T}=\frac{z}{2}\left[x_{\mathrm{A}} \ln \frac{\beta-1+2 x_{\mathrm{A}}}{x_{\mathrm{A}}(\beta+1)}+x_{\mathrm{B}} \ln \frac{\beta-1+2 x_{\mathrm{B}}}{x_{\mathrm{B}}(\beta+1)}\right]
$$

For small $(w / z k T)$, expansion of the exponential, logarithmic and binomial terms up to quadratic terms in both $w$ and $x_{\mathrm{A}} x_{\mathrm{B}}$ yields:

$$
\frac{g^{\mathrm{E}}}{R T}=\frac{w}{k T} x_{\mathrm{A}} x_{\mathrm{B}}\left[1-\frac{1}{2}\left(\frac{2 w}{z k T}\right) x_{\mathrm{A}} x_{\mathrm{B}}-\frac{1}{2}\left(\frac{2 w}{z k T}\right)^{2}+\frac{1}{2}\left(\frac{2 w}{z k T}\right)^{2} x_{\mathrm{A}} x_{\mathrm{B}}-\left(\frac{2 w}{z k T}\right)^{2}\left(x_{\mathrm{A}} x_{\mathrm{B}}\right)^{2}+O^{3}\left(w, x_{\mathrm{A}} x_{\mathrm{B}}\right)\right] \text {. }
$$

The term in square brackets shows contributions to the excess Gibbs energy up to second order both due to large $w$ alone and to large $w$ and $x_{\mathrm{A}} x_{\mathrm{B}}$ combined. That is, even at infinite dilution $\left(x_{\mathrm{B}} \rightarrow 0\right)$ there can be ordering effects, e.g. of solute pairs if $w$ is large.

From thermodynamics,

$$
\begin{aligned}
\ln \gamma_{\mathrm{B}} & =\left(\frac{\partial \frac{n_{\mathrm{T}} g^{\mathrm{E}}}{R T}}{\partial n_{\mathrm{B}}}\right)_{T, P, n_{\mathrm{A}}} \\
& =\frac{w}{k T} x_{\mathrm{A}}^{2}\left\{1-\frac{1}{2}\left(\frac{2 w}{z k T}\right)^{2}+\frac{1}{2}\left(\frac{2 w}{z k T}\right) x_{\mathrm{B}}\left(1-3 x_{\mathrm{A}}\right)-\frac{1}{2}\left(\frac{2 w}{z k T}\right)^{2} x_{\mathrm{B}}\left[1-3 x_{\mathrm{A}}+2 x_{\mathrm{A}} x_{\mathrm{B}}\left(2-3 x_{\mathrm{A}}\right)\right]\right\}
\end{aligned}
$$

$n_{\mathrm{T}}$ is the total number of moles, $n_{\mathrm{T}}=n_{\mathrm{A}}+n_{\mathrm{B}}$. At infinite dilution $x_{\mathrm{A}} \rightarrow 1, x_{\mathrm{B}} \rightarrow 0$, the final result is:

$$
\ln \gamma_{\mathrm{B}}=\frac{w}{k T}\left[1-\frac{1}{2}\left(\frac{2 w}{z k T}\right)^{2}\right]
$$

Replacing this in (15) gives:

$$
\ln x_{\mathrm{B}}=c_{0}-\frac{w}{k T}+\frac{2}{z^{2}}\left(\frac{w}{k T}\right)^{3} .
$$

Since $w$ is negative, this equation shows that the random theory predicts an increase in solubility over the (nearly constant) ideal term $c_{0}$ but, since the last term on the right is cubic, the quasichemical approximation provides a correction which diminishes the solubility at high negative values of $w$ (high $g$ ). This is surprisingly what the data in Figs. 5 and 6 show. The correction depends on $z$, the coordination number of the lattice. If $w$ from (20) is put into (33) the solubility data $\ln x_{\mathrm{B}}$ should be able to be represented by a respective cubic plot against $g$. Indeed, Fig. 6 shows that the data points can very well be simulated by a model curve, cubic in $g$.

Several other theories are known to deal with nonideality of polar solutes in nonpolar solvents. These are the Wilson equation, the NRTL (nonrandom, two-liquid) equation and the UNIQUAC (universal, quasichemical) theory for molecules of different sizes. Details of these theories are found in [1]. 


\section{Conclusion}

A model is proposed to account for the effect of pressure on the solubility of nonpolar and slightly polar solutes in supercritical solvents. The development follows the steps of Schröder, see e.g. [7], but taking into account changes of pressure instead of changes of temperature. In contrast to the ideal temperature effect, which is governed by the enthalpy of fusion, the ideal pressure effect is controlled by the change of volume on fusion, and this is a very small quantity.

According to the present model, the much enhanced solubility observed for solutes near and beyond the critical point is attributed to the attractive interactions between solute and solvent, which are rapidly increas-

[1] J. M. Prausnitz, R. N. Lichtenthaler, and E. Gomes de Azevedo, Molecular Thermodynamics of Fluid-Phase Equilibria, $3^{\text {rd }}$ ed., Prentice Hall PTR, Upper Saddle River 1999.

[2] S. K. Kumar and K. P. Johnston, J. Supercritical Fluids 1, 15 (1988).

[3] T. Moriyoshi, T. Kita, and Y. Uosaki, Ber. Bunsenges. Phys. Chem. 97, 589 (1993).

[4] E. Lippert, Z. Naturforsch. 10a, 541 (1955).

[5] N. Mataga, Y. Kaifu, and M. Koizumi, Bull. Chem. Soc. Jpn. 29, 465 (1956).

[6] W. Liptay, Z. Naturforsch. 20a, 1441 (1965).

[7] K. S. Pitzer, Thermodynamics, $3^{\text {rd }}$ ed., McGraw-Hill, Inc., New York 1995. ing. The solutions are then extremely nonideal in the sense of Raoult's law.

The solvent-solute interaction is modeled according to the theory of Liptay for the effect of solvent on displacement of the absorption maximum of the molecule from the gas state to the solvated state.

\section{Acknowledgements}

G. A. A. wishes to thank the Deutscher Akademischer Austauschdienst (DAAD) for a generous scholarship to stay at the University of Mainz, Germany during the summer of 2002. For providing us with samples of bithiophene we are indebted to Prof. Rüdiger Wortmann $(\dagger)$ from the Universität Kaiserslautern, Germany.

[8] R. T. Kurnik and R. C. Reid, AIChE J. 27, 861 (1981).

[9] C. J.F. Böttcher and P. Bordewijk, Theory of Electric Polarization, Vol. 1, Elsevier, Amsterdam 1973.

[10] F. Neitzel, Doctoral Dissertation, Mainz, Germany (1999).

[11] S. V. Rodrigues, D. Nepomuceno, L. V. Martins, and W. Baumann, Fresenius J. Anal. Chem. 360, 58 (1998).

[12] T. Makita, H. Kubota, Y. Tanaka, and H. Kashiwagi, Refrigeration 52, 543 (1977).

[13] N. Spiliotis, K. Magoulas, and D. Tassios, Fluid Phase Equilibria 102, 121 (1994). 